\title{
Impact de la pollution de temps de pluie sur l'alimentation en eau potable : les moyens de lutte
}

\author{
Louis Herremans \\ Anjou-Recherche - Branche Réseaux - 24 rue Saarinen - SILIC 248 - 94568 RUNGIS Cédex
}

\section{Introduction}

Dans les zones fortements urbanisées, le ruissellement pluvial urbain a été considéré pendant longtemps (et il l'est encore aujourd'hui dans beaucoup de cas) comme un problème essentiellement quantitatif (inondations, débordements de réseaux) contre lequel il fallait se prémunir en évacuant le plus rapidement possible les eaux vers le milieu récepteur.

Cette vision du problème global des eaux de temps de pluie, si elle est nécessaire (les inondations récentes de Nîmes sont là pour le prouver), n'est cependant pas suffisante.

En effet, depuis un peu plus d'une décennie, les études réalisées pour analyser la qualité des eaux rejetées par temps de pluie dans les rivières et leur impact sur celles-ci (notamment sur la production d'eau potable) ont montré que ces eaux constituaient une des sources les plus importantes de dégradation des milieux récepteurs au même titre que les eaux usées.

\section{Comment l'eau de pluie devient un polluant}

Les pollutions rejetées par temps de pluie sont de diverses formes et résultent des différentes étapes auxquelles va être soumise l'eau météorique avant de rejoindre le milieu récepteur, à savoir :

- le lessivage de l'atmosphère ;

- le ruissellement sur les sols imperméabilisés ou non ;

- le transport dans les collecteurs d'assainissement.

Chacune de ces étapes va participer à la pollution rejetée de façon plus ou moins importante. a) Le lessivage de l'atmosphère constitue la partie la plus faible, mais qui, dans certains cas précis, ne pourra être ignorée du fait, en particulier, de la présence des complexes industriels (phénomènes des pluies acides, apports de métaux ou de nutriments).

Ainsi, on a pu constaté, à travers des mesures réalisées sur ces eaux, que les normes fixées pour les eaux destinées à la fabrication d'eau potable pouvaient être largement dépassées, sur les eaux de pluie, pour certains paramètres.

b) Le lessivage des sols par ruissellement constitue la majeure partie de l'apport de la pollution.

En effet, ce lessivage, entraînant les poussières et salissures présentes sur le sol (excréments animaux, résidus de la circulation automobile, produits utilisés dans l'agriculture, ...) va engendrer une pollution aux caractéristiques très variées selon les sites touchés : industriels, urbains, ou agricoles.

Ainsi, certains sites industriels seront générateurs de pollutions essentielleemnt toxiques (micropolluants) et/ou à base de produits pétroliers divers.

Les zones agricoles, par contre, seront à l'origine de la présence de concentrations élevées en nitrates ou en pesticides, dûes aux pratiques agricoles actuelles, dans les rivières ou les nappes souterraines.

Les zones urbaines seront, quant à elles, génératrices essentiellement de pollutions organiques et métalliques (vie urbaine, circulation automobile, déchets divers sur les sols).

En zone littorale, les pollutions du type bactérien ne pourront pas non plus être négligées (en provenance de l'élevage ou de la vie urbaine elle-même, ce type de pollution peut mettre en péril certaines activités telles que le tourisme ou la conchyliculture). 
c) Le transport dans les collecteurs d'assainissement a, quant à lui, plusieurs conséquences sur les rejets de temps de pluie :

- la remise en suspension de sédiments dispersés hors période pluvieuse en fond de collecteur (dans les réseaux unitaires, notamment, réseaux qui forment la majorité du système d'assainissement des centres-villes anciens) et fortement chargés en pollution organique essentiellement ; - le mélange plus ou moins important d'eaux usées et d'eaux de ruissellement (cas des réseaux unitaires) engendrant des déversements pouvant être très chargés en matières organiques et azotées ;

- enfin, le dysfonctionnement éventuel des stations d'épuration des eaux résiduaires du fait d'apports trop importants d'eau pendant les pluies et ayant pour conséquence, bien souvent, des pertes importantes de boues.

On voit donc que les mécanismes engendrant la transformation de l'eau météorique sont très complexes et qu'ils peuvent s'enchaîner les uns aux autres pour constituer globalement un apport de pollution au milieu naturel considérable.

\section{Une pollution de temps de pluie quantitativement importante}

Afin de donner un ordre de grandeur du phénomène pluvial, on peut estimer que, globalement sur l'année, en région parisienne la pollution dûe aux rejets de temps de pluie est environ le double de la pollution permanente (eaux rejetées après traitement en station d'épuraton et eaux non traitées).

Cependant, cette vision du problème est beaucoup trop simpliste du fait que le phénomène "pluie" est un phénomène, d'une part aléatoire, et, d'autre part et surtout, non permanent sur l'année.

Aussi, si la vision globale du problème permet d'avoir quelques ordres de grandeur, il faut surtout s'attacher à l'étude d'événements particuliers, tels que les orages d'été, qui produisent des effets de chocs considérables sur les rivières (le cas des orages survenus à la fin du mois de juin 1990 en région parisienne a montré tout l'impact médiatique que pouvaient avoir de tels événements du fait des très fortes mortalités piscicoles qui se sont alors produites).

Afin d'évaluer ces apports massifs d'eaux polluées dans nos rivières, la Compagnie Générale des Eaux a lancé - ou a participé - à des programmes de mesures visant à caractériser précisément la pollution contenue dans les différentes étapes de dégradation décrites précédemment.

Par ailleurs, du fait de la multiplicité des rejets et du caractère épisodique et aléatoire de la pluie qui rendent la mise en place du programme de mesures sur l'ensemble d'une agglomération hasardeuse et peu réaliste financièrement, la Compagnie a décidé, il y a quelques années déjà, d'investir dans l'élaboration de programmes de calcul informatiques, en collaboration avec l'Agence de l'Eau Seine-Normandie et le Syndicat des Eaux d'Ile-de-France.
Ainsi deux modèles, FLUPOL ET KALPLAN, ont été mis au point et permettent, pour le premier, d'évaluer les flux de pollution rejetés par temps de pluie par un réseau d'assainissement drainant un bassin versant urbain et, pour le second, d'évaluer l'impact de ces rejets sur la qualité d'une rivière.

Ces modèles, maintenant parfaitement opérationnels ont déjà permis, sur certaines agglomérations, de déterminer les aménagements à réaliser sur les réseaux en fonction d'objectifs fixés au milieu récepteur.

\section{Quel est l'impact de ces polluants sur les rivières ?}

L'impact le plus visible (et le plus médiatique, comme nous l'avons souligné précédemment) est constitué par les mortalités piscicoles.

Cependant, si cet impact est l'impact extrême, d'autres conséquences peuvent être également très graves et le seront d'autant plus que la qualité moyenne de la rivière, en temps non pluvieux, sera bonne.

Tel est le cas de la Seine à l'amont de Paris, dont la qualité s'est grandement améliorée depuis la mise en service de la station d'épuration de Valenton, et qui soufre maintenant, en particulier au niveau de la production d'eau potable, de pertubations fréquentes par temps de pluie qui, auparavant, passaient inaperçus du fait d'un " bruit de fond» beaucoup plus important.

Ainsi, on constate, au niveau des différents usines de production d'eau potable (en particulier celles de Choisyle-Roi et Neuilly-sur-Marne), des désordes graves dûs à des rejets importants en période pluvieuse provenant de zones en amont.

Pour exemple, durant l'automne 1991, l'usine de Neuilly-sur-Marne fut arrêtée pendant plusieurs heures à la suite de teneurs considérables d'atrazine dans la Marne (plusieurs centaines de $\mathrm{mg} / \mathrm{l}$ ).

La présence de ce pesticide résultait d'un épisode pluvieux de moyenne importance qui avait provoqué le " rinçage " d'une canalisation dans laquelle se trouvaient des dépôts de ce produit. Dans ce cas précis, la pluie a constitué le facteur déclenchant d'une pollution « accidentelle " (mais de tels accidents sont extrêmement fréquents!).

Aussi, en dehors de ce cas exemplaire, les pertubations les plus fréquentes proviennent du lessivage des sols entraînant de fortes quantités d'hydrocarbures et des surverses de réseaux unitaires provoquant des montées d'ammoniac.

Ces perturbations, si elles entraînent rarement un arrêt complet des usines, engendrent des ralentissements notables de la production de l'usine concernée, des surcoûts importants de réactifs et un pilotage des usines en configuration de crise.

Cependant, au-delà de ces impacts visibles et chiffrables économiquement, la pollution véhiculée par les eaux de temps de pluie engendre une dégradation lente et progres- 
sive de nos rivières et entraîne une modification de l'écosystème qui, dans certains cas, peut devenir irréversible.

Ces phénomènes sont encore très mal connus et nécessitent le passage par les méthodes écotoxicologiques. Ils mobilisent, au niveau européen, les efforts de recherche de nombreux spécialistes tant du domaine public que du domaine privé.

\section{Quelles sont les solutions?}

Contrairement aux eaux usées permanentes dont les variations de débit, au cours de la journée, sont relativement modestes et dont le traitement complet peut être effectué dans des ouvrages d'épuration compacts (telles que les usines de Monaco ou d'Antibes réalisées par la Société OTV), les eaux rejetées par temps de pluie dans les milieux récepteurs correspondent à des variations de débit très rapides et importantes et représentent, sur l'ensemble d'une pluie (car c'est l'ensemble des eaux et non le premier flot d'orage qu'il faut traiter), des volumes considérables (certains déversoirs d'orage de l'agglomération parisienne peuvent rejeter plusieurs centaines de milliers de $\mathrm{m}^{3}$ en quelques heures).

Dans ces conditions, il n'est pas réaliste d'imaginer traiter l'ensemble de ces eaux, sans dispositifs préalables, au niveau d'une station d'épuration.

Aussi, est-il nécessaire, soit, comme le font nos voisins, allemands, de mettre en place des volumes de stockage importants en différents points de réseau, soit d'imaginer des dispositifs de traitement spécifiques.

Les volumes de stockage permettent d'assurer un prétraitement des eaux, (par décantation) et un envoi progressif des eaux vers la station d'épuration après la pluie (tel que le bassin de Cleunay réalisé à Rennes par la Société SOGEA).

Concernant les traitements spécifiques, ceux-ci sont, en priorité, nécessaires pour les réseaux séparatifs (traitement des hydrocarbures, utilisation de la décantation lamellaire), mais également pour les réseaux unitaires pour lesquels les objetifs visés ne permettraient pas d'envisager des volumes de stockage réalistes du fait des tailles nécessaires.

Par ailleurs, comme le prévoit la Directive Européenne sur le traitement des eaux résiduaires de Mai 1991, il sera nécessaire que chaque station d'épuration dispose d'une capacité d'accueil en cohérence avec le traitement des événements pluvieux non exceptionnels.

Enfin, il sera nécessaire de mettre, en place, d'une part, comme le font déjà certains services d'assainissement, une gestion adaptée du réseau d'assainissement afin d'utiliser au mieux la capacité des différents collecteurs et réserver aux pluies exceptionnelles les déversements inévitables tout en les minimisant afin d'éviter des perturbations trop notables au milieu récepteur et, d'autre part, des moyens de traitement des résidus collectés dans les ouvrages spécifiques compatibles avec la politique actuellement très volontariste en matière de déchets.

La mise en place de moyens de traitement adaptés au problème pluvial va, bien évidemment, mobiliser des sommes considérables compte-tenu de l'importance des ouvrages à réaliser (dans le cas de l'agglomération parisienne, plusieurs dizaines de milliards de francs seront probablement nécessaires).

Afin d'essayer de minimiser au maximum les coûts d'investissement et permettre ainsi de répondre aux exigences européennes dans les délais fixés, la Compagnie Générale des Eaux mène actuellement un vaste programme de recherche, en liaison avec les Agences de l'Eau et le ministère de l'Environnement, visant à mettre au point, dans des délais très brefs, d'une part, des techniques de dépollution à la source (nettoyage des voieries, limitation des débits, techniques compensatoires, ...), d'autre part, des ouvrages compacts de traitement spécifiques basés sur la décantation lamellaire et, enfin, des techniques de conditionnement et de traitement des résidus de dépollution.

La prise en compte du problème pluvial est donc une des clés de la restauration des eaux superficielles, toutes les actions de dépollution menées jusqu'à présent risquant de ne pas avoir l'efficacité souhaitée si ce problème n'est pas traité rapidement. Elle va nécessiter un effort important tant au niveau économique qu'au niveau de la recherche de solutions.

C'est au prix de tels efforts que nous pourrons peut-être, dans quelques années, nous réconcilier avec l'eau qui traverse nos villes et ne plus penser à la cacher à tout prix. 